\title{
Hydration induced stress on DNA monolayers
}

\section{grafted on microcantilevers}

Carmen M. Domínguez, Priscila M. Kosaka, Guillermo Mokry, Valerio Pini, Oscar Malvar, Mercedes del Rey, Daniel Ramos, Álvaro San Paulo, Javier Tamayo and Montserrat Calleja

Instituto de Microelectrónica de Madrid, IMM-CNM (CSIC), 28760, Tres Cantos, Spain

Keywords: nanomechanical biosensors, DNA, microcantilever sensors, AFM, hybridization, Selfassembled monolayers, polyelectrolyte

Surface tethered single-stranded DNA films are relevant biorecognition layers for oligonucleotide sequence identification. Also, hydration induced effects on these films have proven useful for the nanomechanical detection of DNA hybridization. Here, we apply nanomechanical sensors and atomic force microscopy to characterize in air and upon varying relative humidity conditions the swelling and deswelling of grafted single stranded and double stranded DNA films. The combination of these techniques validates a two-step hybridization process, where complementary strands first bind to the surface tethered single stranded DNA probes and then slowly proceed to a fully zipped configuration. Our results also demonstrate that, despite the slow hybridization kinetics observed for grafted DNA onto microcantilever surfaces, ex-situ sequence identification does not require hybridization times typically longer than 1 hour, while quantification is a major challenge. 


\section{Introduction}

A variety of nucleic acid biosensors, such as electrochemical, nanomechanical or optical biosensors, rely on single stranded DNA self-assembled monolayers on inorganic surfaces. ${ }^{1,2}$ In depth knowledge about the conformations of such selfassembled monolayers (SAMs) is crucial to understand and control the performance of any surface based nucleic acid sensor. Particularly, the intriguing mechanical properties of these films on microcantilevers have been harnessed to develop labelfree nanomechanical sensors for DNA detection. ${ }^{2}$ The changes in surface stress induced by the hybridization of probe-target strands have been followed in buffer solutions to detect the hybridization in real time and with sufficiently high sensitivity as to avoid the need of pre-amplification steps in complex samples, such as cell lysate. ${ }^{3,4}$ Hydration driven structural changes in DNA have recently gained further attention for the generation of responsive biomaterials. ${ }^{5,6}$ We have also previously demonstrated exsitu hybridization detection in air after incubation with the sample solutions by harnessing the hydration-induced tension in nucleic acid films ${ }^{7}$ or water desorption. ${ }^{8}$ Surface stress biosensors have proven high sensitivity that adds to the advantages of a label-free technology. However, their application to clinical diagnosis will also demand for a method capable of providing hundreds of measurements in a short time, as well as manageable preparation steps for the microcantilever sensors that can still provide reproducible mechanical patterns. ${ }^{9,10}$ All the above reasons highlight the need to pursue in depth understanding of the hybridization process and of the conformations of the DNA layers.

The experimental work carried out during the last decade on DNA SAMs on microcantilever surfaces has revealed that the mechanical signatures of SAMs of single and double stranded DNA (ssDNA and dsDNA) strongly depend on the immobilization protocols used, ${ }^{11,12}$ the gold nanostructure ${ }^{13,14}$ and even the electrostatic 
forces during immobilization. ${ }^{15,16}$ Recent theoretical and experimental work has highlighted the fact that even slightly different experimental conditions, such as ionic strength, temperature or $\mathrm{pH}$ variations during immobilization, may lead to largely divergent mechanical effects. ${ }^{17,18}$ The final strand density in self-assembled monolayers plays a critical role in nanomechanical sensing ${ }^{9}$ and it is indeed far from being fully controllable. ${ }^{19,20-23}$ One singularly limiting difficulty has been to accomplish the anchoring of the ssDNA probes with a well-defined uniform density and a standing up conformation. ${ }^{19,24}$ For such purpose, sophisticated nanografting methods need to be applied. ${ }^{25,26}$ Alternatively, the gold layer needs to comply with very restrictive conditions regarding surface roughness ${ }^{13}$ and surface electrostatics, ${ }^{16}$ while long incubation times are also applied, ${ }^{24}$ which bans the use of technologies for high multiplexing, such as ink-jet low volume dispensing. ${ }^{3,10}$

In this work we study the effect of relative humidity $(\mathrm{RH})$ changes in ssDNA and dsDNA SAMs on gold. Non-ideal DNA SAMs, as those obtained by ink-jet deposition and resulting in a coiled/lying down conformation of the DNA strands are used here. We demonstrate they provide predictable static nanomechanical responses and serve as an efficient bioreceptor layer for nanomechanical sensing. We also follow the dsDNA conformations with $\mathrm{RH}$ for two different hybridization times and two target concentrations.

\section{Results and discussion}

We show here a comprehensive study of the mechanical response of ssDNA monolayers on microcantilevers, as well as those after incubation with their complementary sequences at two different hybridization times and for two target concentrations. For this study we have translated hybridization protocols optimized for well-established fluorescence based molecular biology technologies to the nanomechanical sensing field. The aim is to improve the specificity of the 
nanomechanical assays for nucleic acid detection. Thus, we have chosen PerfectHyb ${ }^{\mathrm{TM}}$ Plus from Sigma-Aldrich, which is a hybridization buffer optimized to yield maximum signal with minimum background. We have also performed the incubation at $30 \stackrel{\circ}{\circ}$, closer to the melting temperature of the hybrids, to avoid cross-hybridization. We have found that for the commercial gold coated microcantilevers and for the buffer solutions and functionalization protocols used in this study (see experimental section), the ssDNA strands are always found in a lying down/coiled conformation when the surfaces are removed from solution to air environment. This is derived from the average $2 \mathrm{~nm}$ height of the monolayers measured by AFM. The methodology for the monolayer height characterization is described in detail in Supplementary Materials section S5. Figure 1 shows the surface stress variation with relative humidity $(R H)$ for 40 microcantilevers functionalized with SsDNA, using the sequence referred to as Oligo1 (see table 1 in the experimental section for details). Five sensor chips with eight microcantilevers per chip were independently functionalized in $\mathrm{TE}-1 \mathrm{M} \mathrm{NaCl}$, as detailed in the experimental section. Clearly, the ssDNA monolayers show highly reproducible surface stress hydration/dehydration patterns. We have also corroborated the high repeatability of the surface stress measurements by measuring eight cantilevers from one functionalized chip after each of 5 consecutive rinsing steps with their corresponding removal and re-alignment of the chip in the optical set-up (curves shown in Supplementary Materials FigureS1). 

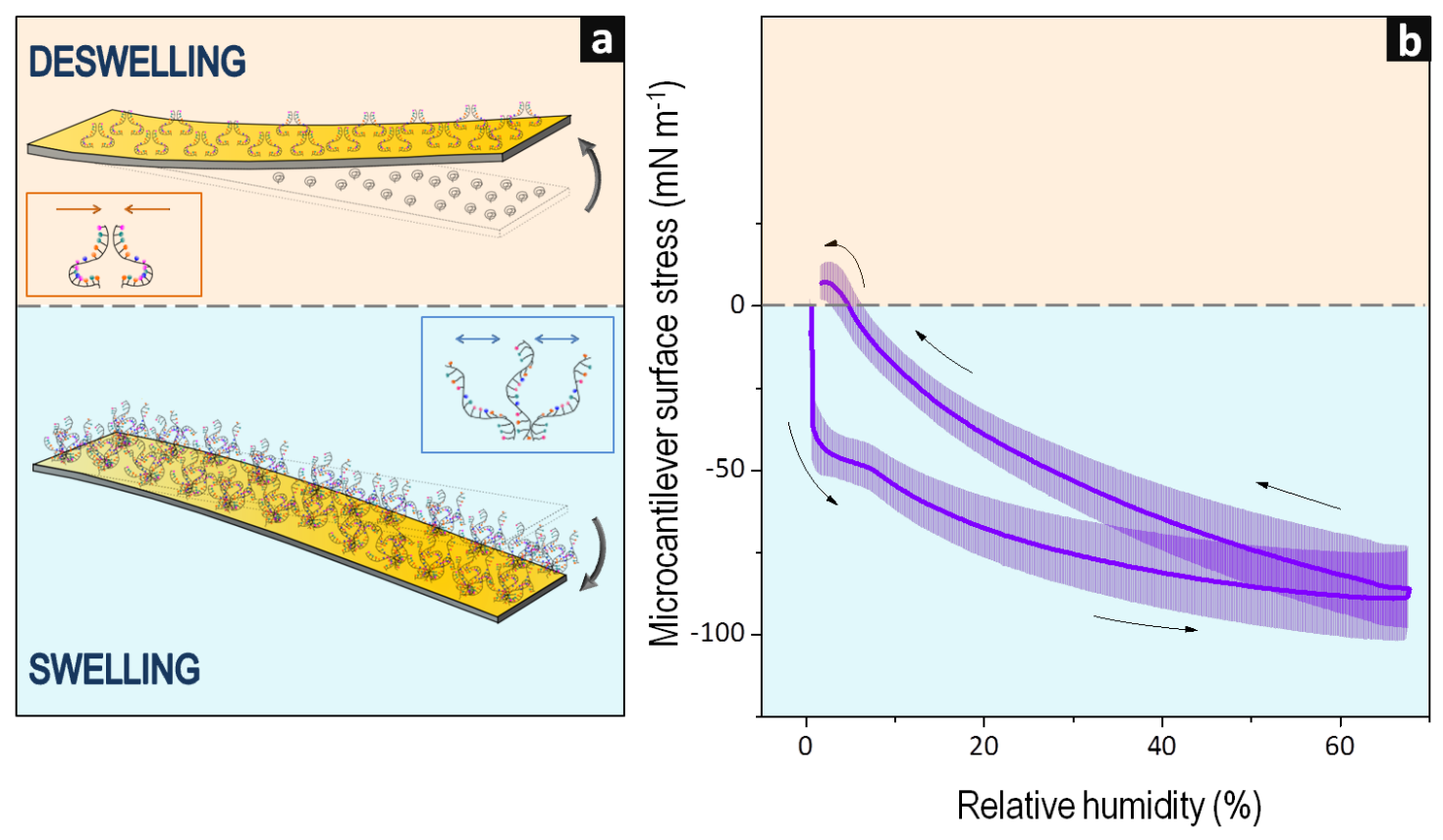

Figure 1 a) Sketch of the swelling and deswelling of surface tethered SsDNA strands on the microcantilever surface. b) Surface stress variation of ssDNA functionalized microcantilevers (sequence Oligo1). The shadowed area represents the standard deviation of the surface stress curves measured in 40 microcantilevers from five independently functionalized chips.

The good reproducibility of the surface stress hydration/dehydration patterns highlights the relevance of evaluating if the coiled conformation, commonly considered as non-ideal for nanomechanical detection, may serve as a reliable bioreceptor layer for label-free DNA sequence identification. We have studied the surface stress hydration/dehydration patterns of microcantilevers functionalized with three different sequences Oligo1, Oligo2 and ControlOligo3 (used as negative control), and those after hybridization, as described in the experimental section.

We study first the surface stress variation of the ssDNA probe layer with varying relative humidity (see figure 1b). The probe layer of SsDNA shows an increase in the compressive stress with increasing relative humidity from $0 \%$ to $70 \%$ that we attribute to the swelling of the polyelectrolyte layer. Since water acts as a solvent for the polyelectrolyte DNA, as $\mathrm{RH}$ rises, the persistence length decreases and ssDNA chains 
explore a wider conformational space. ${ }^{18}$ Hence, the entropy penalty needs to be balanced by a larger bending at increasing relative humidity, ${ }^{11}$ as recorded by our readout instrument. When relative humidity is decreased from $70 \%$ back to $0 \%$ the monolayer shrinks, showing a decrease of the compressive stress until a low humidity around $2-3 \%$ when a peak related to tensile stress in the monolayer arises. Thus, in this dehydration curve, the ssDNA monolayer shrinks the most at the $\mathrm{RH}$ range 2 - 3 $\%$, while it adopts again a more extended conformation at $0 \% \mathrm{RH}$. We attribute this peak to the dominancy of hydration forces over conformational entropy. ${ }^{17,27,28}$ Rabin et al. ${ }^{18}$ explain this tensile stress peak by a model that considers that at low water vapor concentrations (below 2\%) the adsorption of water is accompanied by enhanced expulsion of vacancies, and thus, compression of the monolayer. We must note that the tensile peak appears here (for initially coiled strands in air) in the dehydration curve and not in the hydration curve from $0 \%$ to higher relative humidity, as it is the case studied in the Rabin model and observed experimentally for high density monolayers in a standing up configuration (see Supplementary Materials). These features reveal the complex force landscape involved in the ssDNA swelling and deswelling process. We would also like to point out the fact that equilibrium is not reached during the process, given the rate of change of relative humidity $\left(10.00 \pm 0.08 \% \mathrm{~min}^{-1}\right)$ and the slow kinetics for conformational changes in the SSDNA SAM, that may extend for hours. ${ }^{18,29}$ Thus, we believe that the dominant contribution being that of conformational entropy or hydration forces in the swelling and deswelling dynamics of the ssDNA polyelectrolyte largely depends on the initial density and conformation of the monolayer measured. ${ }^{28,30}$ Based on reference XPS measurements we estimate a surface coverage in the range of $2.5-3.5 \times 10^{13}$ molecules $/ \mathrm{cm}^{2}$ in the present experiments, where the AFM characterization of the surfaces clearly shows a laying down conformation of the ssDNA. Also, we have found that the surface stress curves do not significantly differ for the two sequences studied, despite the difference in length (four bases) and base 
composition. We speculate that small variations in the surface density may hinder the expected increase in the surface stress variation for longer ssDNA chains. ${ }^{11}$ We do not consider the effect of electrostatic interactions in this study, as previous work with uncharged PNA molecules did not show remarkable differences in the surface stress response with relative humidity of this uncharged DNA mimic. ${ }^{7}$

The surface stress variation of ssDNA films as a function of the $\mathrm{RH}$, shown in figure 1, reveals distinct hydration/dehydration features after incubation with the target solution depending on the hybridization conditions. For the sake of simplicity, we will focus from this point onwards only on the swelling curve to study the behavior of the oligonucleotide monolayer after incubation with a target solution.

After incubation of the microcantilevers functionalized with sequence ControlOligo3 (used as negative control), in the solution having no complementary strands to this probe sequence, we do not observe any changes in the surface stress pattern of the monolayer, as measured by the microcantilever bending variations with $\mathrm{RH}$. See figure $2 \mathrm{a}$ and $2 \mathrm{~b}$. Note that we have performed an accurate calibration of the surface stress, as described in the Supplementary Materials. We label this unchanged surface stress pattern as $A$.

After incubation of the microcantilevers functionalized with the probe sequence Oligo1 in the solution containing complementary sequences ComplOligo1 at a concentration of $300 \mathrm{nM}$, we observe two distinct patterns depending on the hybridization time. We see an increase in the surface stress variation upon hydration when incubation time is limited to one hour (figure $2 \mathrm{c}$ ). We label this stress pattern as B. We hypothesize that the hybridization has only partially occurred in most of the strands involved. It is well known that the complementary strand association during hybridization on surfaces proceeds through a complex set of intermediate steps and that surface tethering slows down hybridization kinetics up to three orders of magnitude. ${ }^{31,32}$ Recent theoretical work has advanced our knowledge about the 
complex processes occurring during DNA duplex formation on surfaces. Medalion et al. hypothesize that the kinetic effects of oligonucleotide hybridization on grafted surfaces might result from hindered diffusion of target DNA into networks formed by crosshybridization. ${ }^{33}$ This might as well explain the known lower hybridization efficiency, but higher stability, of the duplex for surface tethered oligonucleotides. ${ }^{31,34,35}$ Thus, the added strands, in a non-zipped configuration, imply a larger configurational entropy penalty, as the non-zipped strands have short persistence length and the increased number of molecules in the same surface area implies a larger surface stress upon the ssDNA polyelectrolyte swelling. Molecular dynamics simulations of DNA hybridization on surfaces have also shown that the cited non-zipped intermediate states occur during the hybridization process. ${ }^{36-38}$

When the incubation with the complementary strands is performed overnight, we find that surface stress variation with increasing humidity (swelling curve) is largely reduced compared to ssDNA stress pattern. (See pattern $C$ in figure $2 d$ ). Double stranded DNA has a much higher persistence length than single stranded DNA (in solution, $L p_{d s D N A}$ $\left.50 \mathrm{~nm}, \mathrm{~L} p_{\text {SSDNA }} \sim 0.75 \mathrm{~nm}\right) .{ }^{11}$ Given this and the short length of the oligonucleotides under study, a minimum swelling is expected for dsDNA when $\mathrm{RH}$ rises from 0 to $70 \%$ and thus, we expect a reduced cantilever bending variation when most of the strands on the SAM have fully hybridized. We have repeatedly found this behavior for probe sequence Oligo1 after overnight $(\mathrm{O} / \mathrm{N})$ incubation with samples containing ComplOligo1 at 300 nM. Surprisingly, most of the chips functionalized with sequence Oligo2 show pattern B instead, even for overnight incubation in $300 \mathrm{nM}$ of complementary strand ComplOligo2. See figure $2 f$. We hypothesize this arises due to slower hybridization kinetics for sequence Oligo2. Hybridization kinetics on surfaces depend on multiple factors, such as probe length, base composition, ${ }^{20,39,40}$ or competition effects. ${ }^{41}$ Even sequences designed to have similar melting temperatures may have hybridization rate constants that may vary by an order of magnitude when probes are immobilized on 
surfaces ${ }^{31}$ and multiple target strands are involved in the hybridization process. ${ }^{41}$ In addition to the kinetics variations due to probe length or sequence composition, we cannot disregard here other sources for slight variations in the hybridization kinetics, as the cited variations in the grafting density ${ }^{28}$ or sequence dependent interstrand crosslinking. ${ }^{33}$

This has important implications when target sequences are used for the quantification of expression levels, regardless the methodology used, as unless equilibrium is reached at very long incubation times, or the hybridization rates for each used sequence are well known, quantification devises impossible. We find the methodology presented here shares the cited challenges with the fluorescent microarrays, ${ }^{34}$ as we will discuss later on.

In order to test the consistency of our assumptions, we have studied the response of both Oligo1 and Oligo2 functionalized cantilevers after $\mathrm{O} / \mathrm{N}$ incubation but with a lower concentration of target to slow down the hybridization. We have consistently found pattern B for both sequences Oligo1 and Oligo2 for a target concentration of $30 \mathrm{nM}$ (see Supplementary Materials S4).

The surface stress measurements have been complemented by AFM characterization of the biolayers at $0 \% \mathrm{RH}$, after hydration to $37 \% \mathrm{RH}$ and after drying the surface back to $0 \% \mathrm{RH}$. Gold coated silicon surfaces for AFM characterization were prepared in parallel to the microcantilevers, as described in the experimental section. AFM characterization of the SAMs of SSDNA indicates they swell when relative humidity is increased in air from $0 \%$ to $37 \%$, with an increase in the measured height by AFM of $0.230 \mathrm{~nm} \pm 0.035 \mathrm{~nm}$. The layer shrinks and the height goes back to the initial $2.051 \pm 0.025 \mathrm{~nm}$ value when the environment is dried again to $0 \% \mathrm{RH}$. Consistently with the surface stress measurements showing pattern $A$, the height variation remains the same after overnight incubation of the surfaces immobilized with 
sequence ControlOligo3 (negative control) in the sample containing noncomplementary strands. The initial behavior of the ssDNA layer changes when the ssDNA SAM is incubated with solutions containing fully complementary sequences at $300 \mathrm{nM}$ concentration. We again observe two different behaviors depending on the incubation time and on the immobilized sequence. The height increases (monolayer swelling) the most for samples prepared in parallel to those showing surface stress pattern B. Samples immobilized with Oligo1 and incubated for 1 hour with ComplOligo1 and those immobilized with Oligo2 at both hybridization times, give an average height variation of $0.290 \pm 0.014 \mathrm{~nm}$. Consistently with the surface stress measurements, the height measured by AFM remains constant with $\mathrm{RH}$ variations for samples prepared in parallel to those giving surface stress pattern $\mathrm{C}$. This is, Oligo1 functionalized cantilevers after overnight hybridization at $300 \mathrm{nM}$ give and average height variation within the measurement error, of $0.035 \pm 0.035 \mathrm{~nm}$. We interpret that most of the strands on the surface have adopted a fully zipped conformation. The increased rigidity and larger persistence length of the fully zipped dsDNA implies a lower extension of the dsDNA strands when humidity increases from $0 \%$ to larger relative humidity, and thus, a negligible variation in AFM height. 


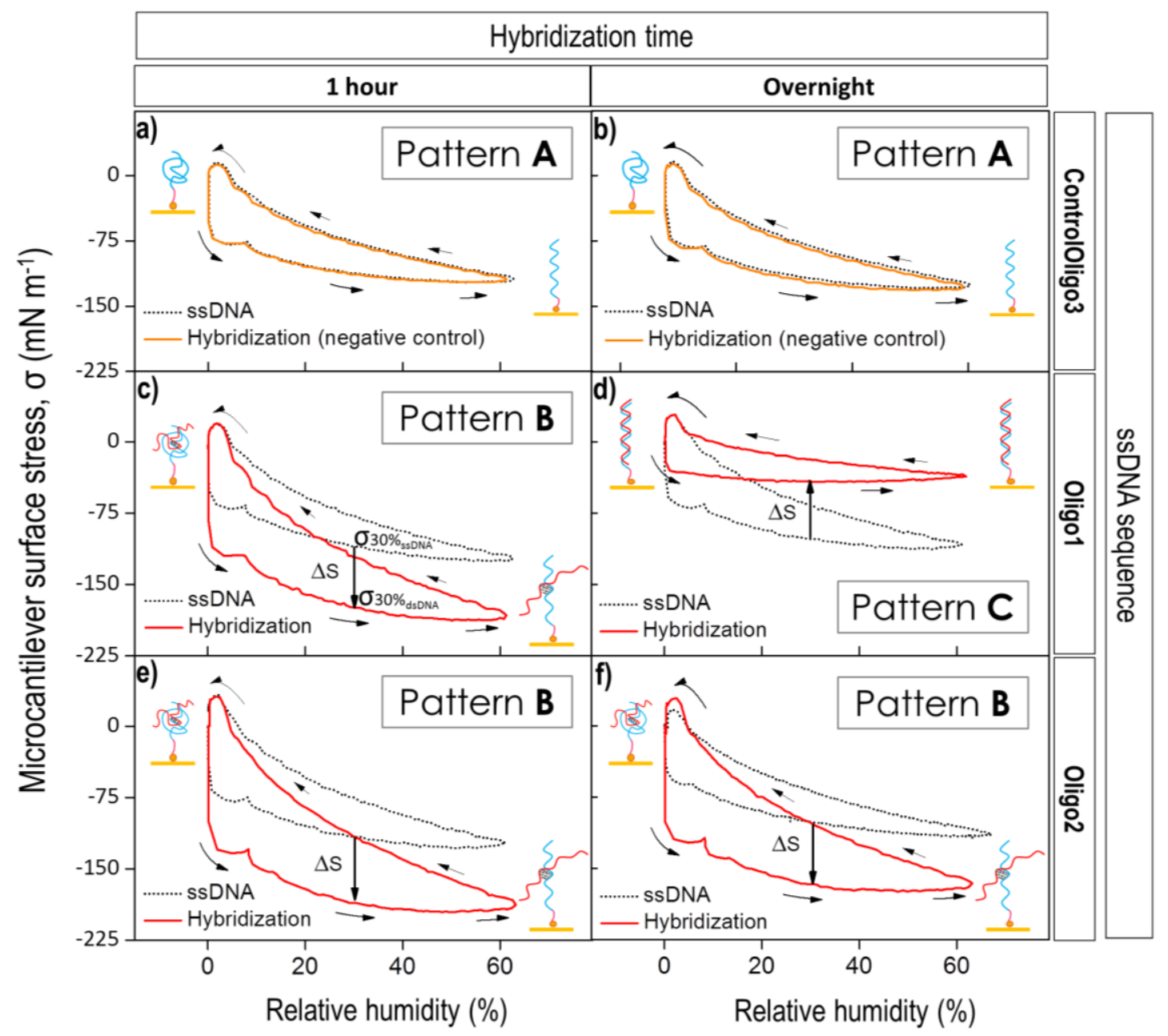

Figure 2. Distinct surface stress hydration/dehydration patterns found after hybridization of the functionalized microcantilevers in different incubation conditions. a) Representative surface stress curve for changing relative humidity of the ssDNA functionalized cantilever (sequence ControlOligo3) before (black dotted line) and after (orange solid line) incubation for 1 hour with the sample solution containing no complementary sequences to the surface probe. We label this surface stress pattern as $A$. b) Same as a) but for overnight $(\mathrm{O} / \mathrm{N})$ incubation. c) Surface stress curve for changing relative humidity before (black dotted line) and after (red solid line) incubation for one hour of the cantilever functionalized with Oligo1 sequence with the sample solution containing complementary strands to the surface probe at a concentration of $300 \mathrm{nM}$. We find that the surface stress variation with increasing relative humidity increases after hybridization. We label this surface stress pattern as B. d) Same as c) for overnight incubation. We find that the surface stress variation with increasing $\mathrm{RH}$ flattens after hybridization. We label this surface stress pattern as C. e) Surface stress curve for changing relative humidity before 
(black dotted line) and after (red solid line) incubation for one hour of the cantilever functionalized with Oligo2 sequence with the sample solution containing complementary strands to the surface probe at a concentration of $300 \mathrm{nM}$. f) Same as d) for overnight incubation. Microcantilevers functionalized with sequence Oligo2 show pattern $\mathrm{B}$ after $\mathrm{O} / \mathrm{N}$ incubation, indicating hybridization kinetics is sequence dependent. The depictions shown on the insets illustrate our hypothesis about the conformational changes giving rise to each surface stress pattern.

Based on the previous study of the different surface stress patterns after hybridization, we arbitrarily define our sensor response signal, $\Delta S$, as the absolute value of the difference in surface stress at $30 \% \mathrm{RH}$ for the swelling curve, before and after incubation with the target sample solution, $\Delta S=\left.\right|_{\sigma_{30 \%, s s D N A}}-\sigma_{30 \%, d s D N A} l$, (see $\Delta S$ in figure 2). We have intentionally chosen the absolute value of the signal to account for both the typical response for short incubation times (pattern B), as well as pattern $C$ for the fully hybridized films. The inclusion of pattern B as a positive detection signal is relevant, as a fast response time for a biosensor is key for its application in diagnosis.

In order to investigate if $\Delta S$ can be used as a reliable sensor signal for sequence specific detection, we have studied 24 chips (192 microcantilevers) in diverse conditions (See Experimental Section). We present the results for the 177 measured microcantilevers in figure S7 in Supplementary Materials, as 15 cantilevers broke during the experiments. Each experiment comprises three different sequences immobilized in three chips with eight cantilevers each. The three chips, two measurement chips and one control for each experiment, were incubated for $1 \mathrm{~h}$ or overnight $(\mathrm{O} / \mathrm{N})$ at $30^{\circ} \mathrm{C}$ in aliquots of the same sample solution containing fully complementary strands to sequences Oligo1 and Oligo2 (ComplOligo1 and ComplOligo2), and a competing unrelated strand (InterferingSeq). See Table 1 in Experimental Section for base composition of each sequence. The concentrations used 
are $30 \mathrm{nM}$ and $300 \mathrm{nM}$, as indicated in figure 3. We depict the average value and standard deviation of the sensor response signal $\Delta S$ for the conditions under study. We also show the average value and standard deviation of $\Delta S$ for the 64 control microcantilevers used. By defining a threshold value for the signal $\Delta S$ as the mean value of the control plus twice the standard deviation, sensitivity is of 0.95 and specificity of 0.98 , defined as the rate of true positives in the assay and true negatives in the controls, respectively; demonstrating the suitability of the methodology for oligonucleotide sequence identification.

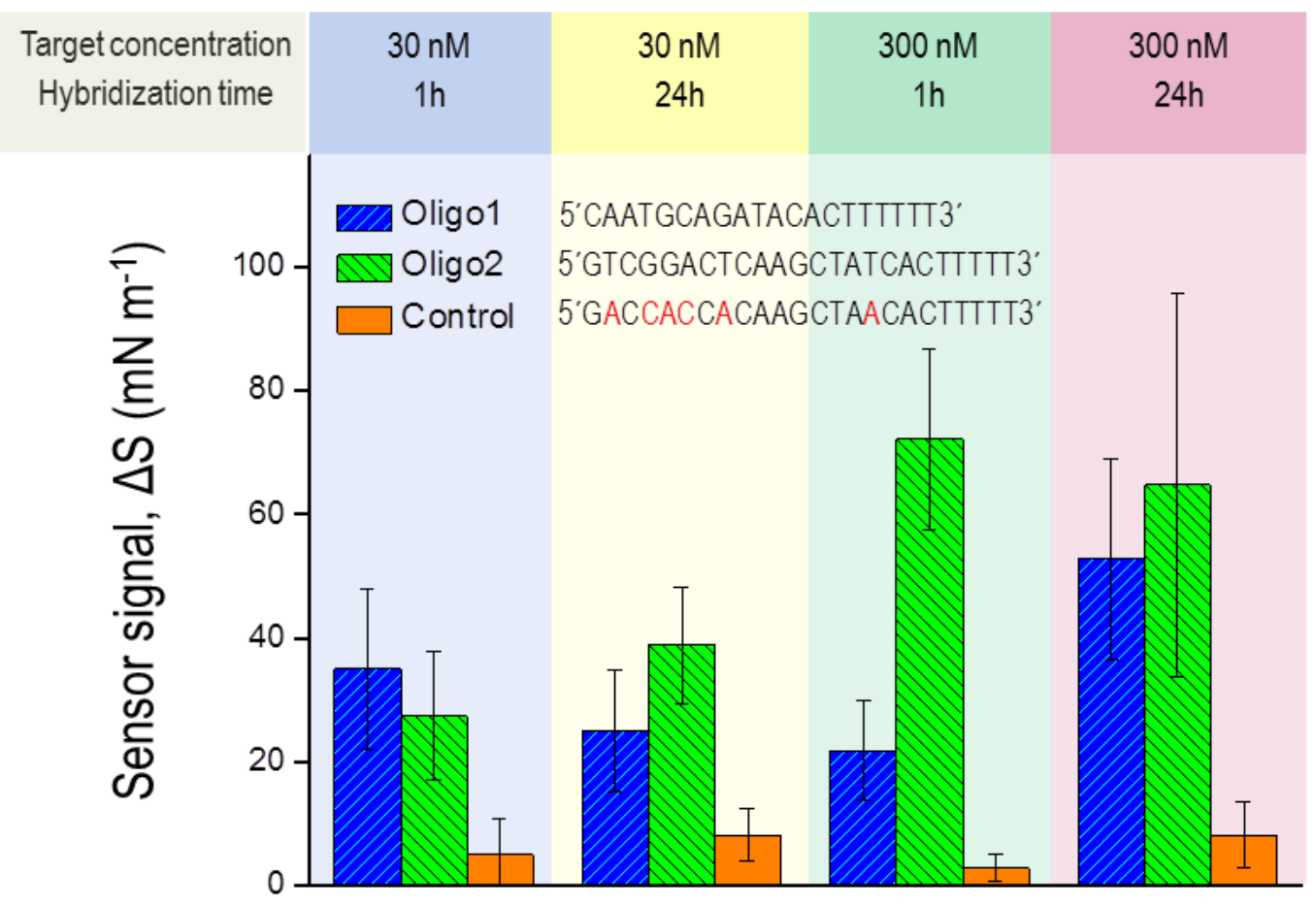

Figure 3. Mean and standard deviation of the sensor signal, $\Delta S$, defined as the absolute value of the surface stress difference at $30 \% \mathrm{RH}$ before and after hybridization, $\quad \Delta S=\left.\right|_{\sigma_{30 \%, S s D N A}}-\sigma_{30 \%, d s D N A} \mid$, measured in 177 microcantilevers functionalized with sequences Oligo1 (blue columns), Oligo2 (green columns) and ControlOligo3 (orange columns). Hybridization experiments were performed for two 
different incubation times ( 1 hour and overnight) and for two target concentrations (30 $\mathrm{nM}$ and $300 \mathrm{nM})$.

However, our results also point out that the signal $\Delta S$ is very sensitive to the experimental conditions discussed above, as seen by the differences in its average value and standard deviation for the experiments performed. Also, $\Delta S$ shows large deviations not only among the two different sequences, but also in experiments performed with the same sequence and in identical incubation conditions (see error bars in green column for Oligo 2 at $300 \mathrm{nM}$ and $24 \mathrm{~h}$ incubation time in figure 3 ). Thus, quantification of the concentration of complementary strands in the solution is not possible with the present methodology. Quantification will require a better control of the immobilization and hybridization conditions, even if a chip is used as a control reference. Other methodologies based on surface grafted DNA, such as fluorescent microarrays, have shown variations of up to $20 \%$ in the measurement of expression levels, even when they were performed by the same user, equipment and in the same conditions. $^{34}$

Previous theoretical works reveal that small amounts of disorder in the DNA monolayer can affect cantilever deflection. ${ }^{28,42-44}$ Moreover, Kosaka et al. ${ }^{19}$, proved the heterogeneity of highly packed DNA monolayers on gold obtained by selfassembly. We speculate that the observed variations in the value of $\Delta S$ from sample to sample, for the same sequence and incubation conditions, could arise due to slight probe density differences or inhomogeneities. These differences may arise from uncontrolled variations in the amount of disulfide groups formed within the thiolated DNA aliquots, that may arise due to several reasons: firstly, thiol groups oxidize over time, even when keeping thiolated DNA aliquots freezed; secondly, since buffer degassing is done at room temperature (note that the concentration of dissolved gas in water is temperature dependent), it is difficult to have a strict control of the dissolved 
oxygen removal in the immobilization buffer; and, finally, oxygen dissolves again in the buffer during overnight probe immobilization in agitation. We have previously demonstrated the effect of thiol or disulfide attachment over the strand density and surface stress on microcantilevers, and demonstrated that thiol grafting produces a larger surface stress, related to higher strand densities, while disulfides produce less dense monolayers and lower surface stress upon grafting. ${ }^{45}$

\section{Conclusions}

We have found that hybridization kinetics and their dependency on base composition play a relevant role in the sequence identification through ex-situ nanomechanical detection. For the DNA monolayers studied, that are far from a standing up brush configuration, different conformations are found for the hybridized layers that arise due to a slow hybridization mechanism on surfaces, in which multiple nucleation events are involved, followed by definitive helix zipping up. This work reveals that these hybridization phases lead to significant qualitative differences on the sensor nanomechanics. The gained knowledge about the grafted monolayers has allowed DNA sequence identification in less stringent conditions for the functionalized microcantilevers. We demonstrate identification of DNA strands for short incubation times $(1 \mathrm{~h})$ and concentrations as low as $30 \mathrm{nM}$. However, quantification of the target DNA concentration is challenging for this ex-situ label-free methodology. We expect the observed variability in the sensing signal value might as well be managed by a better control of the initial gold surface, improved buffer degassing to avoid thiol oxidation, tailored blocking steps capable of better tuning the initial conformation of the SAM layer with high reproducibility or the use of alternative chemistries, such as silanization. Accurate knowledge of the hybridization rates for different probe sequences would also allow quantification by background substraction or corrections similar to those performed in fluorescent based microarrays. ${ }^{46}$ 
In our experiments with 177 microcantilevers, we found 1 false positive out of 64 negative controls and 6 false negatives out of 113 positive samples, which proves exsitu nanomechanical detection as a promising method for high throughput and large multiplexing oligonucleotide sequence identification by hybridization.

\section{Supporting Information.}

S1. Repeatability of the surface stress measurements

S2. Surface stress hydration/dehydration patterns for two different initial conformations of the ssDNA monolayer

S3. Surface stress hydration/dehydration measurements of different DNA monolayers in the same array

S4. Surface stress patterns for low target concentration

S5. AFM measurements

S6. Surface stress response of dsDNA monolayers over hybridization time

S7. Raw data of the sensor signal values

S8. General procedure for the calibration of the experimental setup

This material is available free of charge via the Internet at http://pubs.acs.org.

\section{Corresponding Author}

*mcalleja@imm.cnm.csic.es

\section{Author Contributions}


The manuscript was written through contributions of all authors. All authors have given approval to the final version of the manuscript.

\section{ACKNOWLEDGMENTS}

This work was supported by the ERC Starting Grant "NANOFORCELLS" (ERC-StG2011-278860) and projects INMUNO-SWING ITP-2011-0821-010000, FORCEFORFUTURE CSD2010-00024 and MAT2012-36197.

\section{Experimental Section}

DNA sequences: All thiol modified single stranded DNA and the complementary/non complementary strands (table 1) were purchased from Stab Vida (Caparica, Portugal).

\begin{tabular}{ccc}
\hline Strand name & Type of sequence & Strand Sequence \\
\hline Oligo1 & Probe & $5^{\prime}$-CAATGCAGATACACTTTTTT- ${ }_{3} \mathrm{H}_{6}-\mathrm{SH}_{-} 3^{\prime}$ \\
Oligo2 & Probe & $5^{\prime}$-GTCGGACTCAAGCTATCACTTTTT- ${ }_{3} \mathrm{H}_{6}-\mathrm{SH}_{-} 3^{\prime}$ \\
ControlOligo3 & Negative control & $5^{\prime}$-GACCACCACAAGCTAACACTTTTT- ${ }_{3} \mathrm{H}_{6}-\mathrm{SH}_{-} 3^{\prime}$ \\
ComplOligo1 & Target & $5^{\prime}$-AGTGTATCTGCATTG-3' \\
ComplOligo2 & Target & $5^{\prime}$-GTGATAGCTTGAGTCCGAC-3' \\
InterferingSeq & Unrelated sequence & $5^{\prime}$-GACACTGACATGCCA-3' \\
\hline
\end{tabular}

\section{Table 1. DNA sequences}

The thiolated sequences have a tail of 5 thymines between the $\mathrm{C}_{3} \mathrm{H}_{6}$ spacer and the sequence of interest in order to keep the hybridization bases away from the gold surface, due to the low affinity for gold of thymine. A higher hybridization efficiency is expected for this configuration. ${ }^{47}$

Reagents: toluene, methanol, tris-ethylenediaminetetraacetic acid (Tris-EDTA), sodium chloride ( $\mathrm{NaCl}), 20 x$ saline sodium citrate (SSC), hydrochloric acid $(\mathrm{HCl}), 10 \%$ sodium 
dodecyl sulfate (SDS), commercial $2 x$ prehybridization solution, commercial hybridization buffer PerfectHyb ${ }^{\mathrm{TM}}$ Plus Hybridization Buffer, $\mathrm{NaBH}_{4}$ and ethanol were purchased from Sigma Aldrich (St. Louis, USA). Sulfuric acid $\left(\mathrm{H}_{2} \mathrm{SO}_{4}\right)$ and hydrogen peroxide $\left(\mathrm{H}_{2} \mathrm{O}_{2}\right)$ were also used and were obtained from Panreac (Barcelona, Spain).

Buffers and solutions: All buffers were prepared using molecular biology grade water. The immobilization buffer consisted on 1x Tris-EDTA (which consists in $10 \mathrm{mM}$ Tris$\mathrm{HCl}$ and $1 \mathrm{mM}$ disodium EDTA) with $\mathrm{NaCl} 1 \mathrm{M}$ and $\mathrm{pH}$ 7.5. In order to remove the dissolved oxygen, and thus minimize thiol oxidation, the buffer was degassed by simultaneous sonication and bubble extraction with a vacuum pump for 20 min. Thiolated DNA aliquots were prepared with the degassed 1x TE-NaCl $1 \mathrm{M}$ buffer. Low and high stringency wash buffers contained $0.1 \%$ SDS and 2X SSC and 0.5X SSC, respectively. 1x SSC buffer was prepared by diluting 20x SSC in water.

Surface functionalization: Arrays of eight silicon microcantilevers with $20 \mathrm{~nm}$ gold coating were purchased from Concentris (Basel, Switzerland). The cantilevers are 500 $\mu \mathrm{m}$ long, $100 \mu \mathrm{m}$ wide and $1 \mu \mathrm{m}$ thick. Prior to use, cantilever arrays were deeply cleaned by two consecutive procedures that remove organic contaminants. They were immersed in toluene, methanol and deionized water; dried under a stream of dry nitrogen and irradiated in a UV-Ozone cleaner for 1 hour. Then, the corresponding thiolated DNA was diluted in the immobilization buffer to a final concentration of $5 \mu \mathrm{M}$. The cantilever arrays were incubated in each DNA solution overnight at $25^{\circ} \mathrm{C}$. Afterwards, the arrays were cleaned with low and high stringency hybridization wash buffers to wash out the physisorbed DNA away from the micro cantilever surface and finally rinsed with plenty of Milli-Q water. Cleansing steps were carried out at $25{ }^{\circ} \mathrm{C}$ as well.

Equivalent gold coated silicon surfaces were used for the AFM experiments. Samples were cut in $5 \mathrm{~mm}^{2}$ squares from a silicon wafer. The squares were cleaned in piranha 
solution (piranha solution is highly corrosive and must be handled with extreme caution), extensively rinsed with water and dried under nitrogen. Silicon pieces were coated by e-beam evaporation with a $20 \mathrm{~nm}$ gold layer on top of a $2 \mathrm{~nm}$ adhesion layer of chromium at a deposition rate of $0.02 \mathrm{~nm} \mathrm{~s}^{-1}$. The gold coated squares were cleaned as described before for the gold coated microcantilevers and the protocol followed for the gold surface functionalization was the same as the one used for the microcantilever arrays.

We have been able to re-use gold coated microcantilevers that had been previously functionalized with thiolated DNA strand by following the procedure described by $\mathrm{M}$. Yuan et al. ${ }^{48,49}$ Prior to surface detachment of the thiolated DNA, we cleaned the chips in piranha solution to remove any physisorbed organic compound derived from prehybridization and hybridization buffers. Then, the chips were immersed in $0.5 \mathrm{M}$ $\mathrm{NaBH}_{4}$ solution in 1:1 $\mathrm{H}_{2} \mathrm{O}: \mathrm{EtOH}$ (caution, $\mathrm{NaBH}_{4}$ reacts with $\mathrm{H}_{2} \mathrm{O}$ and releases hydrogen gas) for $2 \mathrm{~h}$. Afterwards, they were rinsed with ethanol and Milli-Q water and ready for use. Re-used microcantilevers showed equivalent responses to new ones.

Surface blocking: Prior to hybridization, the voids on the surface of the functionalized microcantilever chips and on the samples for AFM inspection were blocked by incubation in a $1 \mathrm{x}$ prehybridization solution (Sigma-Aldrich) for 30 minutes at the hybridization temperature $\left(30^{\circ} \mathrm{C}\right)$. The excess of blocking agent was removed by incubating the samples in SSC $1 \times$ buffer for 15 minutes at $25^{\circ} \mathrm{C}$. Then, the samples were rinsed with Milli-Q water.

Target preparation: Complementary sequences to probe oligonucleotides were diluted directly to the corresponding concentration in PerfectHyb ${ }^{\mathrm{TM}}$ Plus Hybridization Buffer (Sigma-Aldrich) and mixed vigorously to homogenize the solution.

Measurement setup: The experiments were performed both in a SCALA-Bio platform (MecWins, Spain) where read-out was made directly from a 96-well plate coated with 
polydimethylsiloxane (PDMS) to attach the arrays to its surface, and also in a similar home-built equipment with capability for read-out of single chips of eight cantilevers. In both instruments, the read-out of the microcantilever deflections is based in the automated two-dimensional scanning of a laser beam across the surface of each microcantilever, and the collection of the reflected beam on the surface of a twodimensional position sensing linear detector (PSD) orthogonally oriented to the reflected beam. Both systems were equipped with an environmental chamber with capability to keep temperature at $25.00 \pm 0.02{ }^{\circ} \mathrm{C}$ degrees and to change relative humidity at a rate of $10.00 \pm 0.08 \% \mathrm{~min}^{-1}$. Prior to the measurement of the surface stress, the cantilevers were equilibrated at $0 \%$ relative humidity under a flow of dry nitrogen for one hour. The systems were calibrated as described in the Supplementary Materials in order to attain accurate values of the surface stress.

Hybridization experiments: In one set of experiments, we incubated three different chips, each containing eight microcantilevers functionalized with one of the three sequences (Oligo1, Oligo2 and ControlOligo3) in three incubation wells filled with aliquots of the same sample solution. The sample contains the two fully complementary strands to sequence Oligo1 and sequence Oligo2 (ComplOligo1 and ComplOligo2), and a competing strand non-complementary to any of the three probe sequences (InterferingSeq). Note that we have chosen two sequences that differ only in 6 bases (see Oligo2 and ControlOligo3 in table 1) and used one of them as negative control, in order to test the specificity of the assay. The response of each microcantilever before and after incubation with the problem solution was recorded. This response comparison serves as an excellent reference to filter out any spurious mechanical signals and deviations in the response between cantilevers with different mechanical properties. 
In a different set of experiments, we immobilized the three probe strands (Oligo1, Oligo2 and ControlOligo3) in alternate cantilevers on the same cantilever array chip by inkjet deposition with sciFLEXARRAYER (Scienion AG, Germany), while leaving one blank microcantilever between the functionalized ones to avoid cross contamination (see Supplementary Materials S4). We then incubate the full chip in the sample solution containing ComplOligo1, ComplOligo2 and InterferingSeq in a single well, wash and dry the chip after reaching the hybridization time in the experiment and measure all cantilevers simultaneously. ${ }^{50}$ We found consistent results for the two measurement methodologies.

Hybridization conditions: The hybridization solution consisted of a mix $1: 1: 1$ of the corresponding complementary strands and non-complementary sequence. Hybridization was performed at $30{ }^{\circ} \mathrm{C}$ for two different incubation times: 1 hour and overnight; at final concentrations of $30 \mathrm{nM}$ and $300 \mathrm{nM}$. At $30{ }^{\circ} \mathrm{C}$, the hybridization temperature was well below the melting temperature for every complementary DNA duplex $\left(37-45^{\circ} \mathrm{C}\right)$, as indicated by the manufacturer of the buffer PerfectHyb ${ }^{\mathrm{TM}}$ Plus Hybridization Buffer (Sigma-Aldrich) for our hybridization conditions. After hybridization, cantilever arrays and AFM samples were cleaned with low and high stringency hybridization wash buffers and extensively rinsed with plenty of Milli-Q water. The higher stringency wash was carried out at the hybridization temperature.

1. Arya, S. K.; Solanki, P. R.; Datta, M.; Malhotra, B. D. Recent advances in selfassembled monolayers based biomolecular electronic devices. Biosensors and Bioelectronics 2009, 24 (9), 2810-2817.

2. Tamayo, J.; Kosaka, P. M.; Ruz, J. J.; San Paulo, A.; Calleja, M. Biosensors based on nanomechanical systems. Chemical Society Reviews 2013, 42 (3), 1287-1311. 3. Zhang, J.; Lang, H.; Huber, F.; Bietsch, A.; Grange, W.; Certa, U.; McKendry, R.; Güntherodt, H. J.; Hegner, M.; Gerber, C. Rapid and label-free nanomechanical 
detection of biomarker transcripts in human RNA. Nature nanotechnology 2006, 1 (3), 214-220.

4. Huber, F.; Lang, H.; Backmann, N.; Rimoldi, D.; Gerber, C. Direct detection of a BRAF mutation in total RNA from melanoma cells using cantilever arrays. Nature nanotechnology 2013, 8 (2), 125-129.

5. Cheng, W.; Hartman, M. R.; Smilgies, D. M.; Long, R.; Campolongo, M. J.; Li, R.; Sekar, K.; Hui, C. Y.; Luo, D. Probing in Real Time the Soft Crystallization of DNA-Capped Nanoparticles. Angewandte Chemie 2010, 122 (2), 390-394.

6. Neumann, T.; Gajria, S.; Bouxsein, N. F.; Jaeger, L.; Tirrell, M. Structural responses of DNA-DDAB films to varying hydration and temperature. Journal of the American Chemical Society 2010, 132 (20), 7025-7037.

7. Mertens, J.; Rogero, C.; Calleja, M.; Ramos, D.; Martin-Gago, J. A.; Briones, C.; Tamayo, J. Label-free detection of DNA hybridization based on hydration-induced tension in nucleic acid films. Nature Nanotechnology 2008, 3 (5), 301-307.

8. Cagliani, A.; Kosaka, P.; Tamayo, J.; Davis, Z. J. Monitoring the hydration of DNA self-assembled monolayers using an extensional nanomechanical resonator. Lab on a Chip 2012, 12 (11), 2069-2073.

9. Kosaka, P. M.; Tamayo, J.; Ruz, J. J.; Puertas, S.; Polo, E.; Grazu, V.; Jesús, M.; Calleja, M. Tackling reproducibility in microcantilever biosensors: a statistical approach for sensitive and specific end-point detection of immunoreactions. Analyst 2013, 138 (3), 863-872.

10. Calleja, M.; Kosaka, P. M.; San Paulo, Á.; Tamayo, J. Challenges for nanomechanical sensors in biological detection. Nanoscale 2012, 4 (16), 4925-4938.

11. Wu, G.; Ji, H.; Hansen, K.; Thundat, T.; Datar, R.; Cote, R.; Hagan, M. F.; Chakraborty, A. K.; Majumdar, A. Origin of nanomechanical cantilever motion generated from biomolecular interactions. Proceedings of the National Academy of Sciences 2001, 98 (4), 1560-1564.

12. Zhang, J.; Lang, H. P.; Yoshikawa, G.; Gerber, C. Optimization of DNA Hybridization Efficiency by pH-Driven Nanomechanical Bending. Langmuir 2012, 28 (15), 6494-6501.

13. Mertens, J.; Calleja, M.; Ramos, D.; Taryn, A.; Tamayo, J. Role of the gold film nanostructure on the nanomechanical response of microcantilever sensors. Journal of applied physics 2007, 101 (3), 034904-034904-8.

14. Tabard-Cossa, V.; Godin, M.; Burgess, I. J.; Monga, T.; Lennox, R. B.; Grütter, P. Microcantilever-based sensors: effect of morphology, adhesion, and cleanliness of the sensing surface on surface stress. Analytical chemistry 2007, 79 (21), 8136-8143.

15. Wong, I. Y.; Melosh, N. A. An Electrostatic Model for DNA Surface Hybridization. Biophysical Journal 2010, 98 (12), 2954-2963.

16. Arroyo-Hernández, M.; Tamayo, J.; Costa-Krämer, J. L. Stress and DNA Assembly Differences on Cantilevers Gold Coated by Resistive and E-Beam Evaporation Techniques. Langmuir 2009, 25 (18), 10633-10638.

17. Zhang, N. H.; Tan, Z. Q.; Li, J. J.; Meng, W. L.; Xu, L. W. Interactions of single-stranded DNA on microcantilevers. Current Opinion in Colloid \& Interface Science 2011, 16 (6), 592-596.

18. Wagman, M.; Medalion, S.; Rabin, Y. Anomalous Swelling of Polymer Monolayers by Water Vapor. Macromolecules 2012, 45 (23), 9517-9521.

19. Kosaka, P. M.; Gonzalez, S.; Martinez, C.; Cebollada, A.; San Paulo, A.; Calleja, M.; Tamayo, J. Atomic force microscopy reveals two phases in single stranded DNA self-assembled monolayers. Nanoscale 2013. 
20. Petrovykh, D. Y.; Perez-Dieste, V.; Opdahl, A.; Kimura-Suda, H.; Sullivan, J.; Tarlov, M. J.; Himpsel, F.; Whitman, L. J. Nucleobase orientation and ordering in films of single-stranded DNA on gold. Journal of the American Chemical Society 2006, 128 (1), 2-3.

21. Petrovykh, D. Y.; Kimura-Suda, H.; Tarlov, M. J.; Whitman, L. J. Quantitative characterization of DNA films by X-ray photoelectron spectroscopy. Langmuir 2004, 20 (2), 429-440.

22. Petrovykh, D. Y.; Kimura-Suda, H.; Whitman, L. J.; Tarlov, M. J. Quantitative analysis and characterization of DNA immobilized on gold. Journal of the American Chemical Society 2003, 125 (17), 5219-5226.

23. Murphy, J. N.; Cheng, A. K. H.; Yu, H. Z.; Bizzotto, D. On the nature of DNA self-assembled monolayers on Au: measuring surface heterogeneity with electrochemical in situ fluorescence microscopy. Journal of the American Chemical Society 2009, 131 (11), 4042-4050.

24. Mertens, J.; Rogero, C.; Calleja, M.; Ramos, D.; Martín-Gago, J. A.; Briones, C.; Tamayo, J. Label-free detection of DNA hybridization based on hydration-induced tension in nucleic acid films. Nature nanotechnology 2008, 3 (5), 301-307.

25. Bosco, A.; Bano, F.; Parisse, P.; Casalis, L.; DeSimone, A.; Micheletti, C. Hybridization in nanostructured DNA monolayers probed by AFM: theory versus experiment. Nanoscale 2012, 4 (5), 1734-1741.

26. Mirmomtaz, E.; Castronovo, M.; Grunwald, C.; Bano, F.; Scaini, D.; Ensafi, A. A.; Scoles, G.; Casalis, L. Quantitative Study of the Effect of Coverage on the Hybridization Efficiency of Surface-Bound DNA Nanostructures. Nano Letters 2008, 8 (12), 4134-4139.

27. Dobrynin, A. V.; Rubinstein, M. Theory of polyelectrolytes in solutions and at surfaces. Progress in Polymer Science 2005, 30 (11), 1049-1118.

28. Hagan, M. F.; Majumdar, A.; Chakraborty, A. K. Nanomechanical Forces Generated by Surface Grafted DNA. The Journal of Physical Chemistry B 2002, 106 (39), 10163-10173.

29. Schwartz, D. K. Mechanisms and kinetics of self-assembled monolayer formation. Annual Review of Physical Chemistry 2001, 52 (1), 107-137.

30. Tanchak, O. M.; Barrett, C. J. Swelling dynamics of multilayer films of weak polyelectrolytes. Chemistry of materials 2004, 16 (14), 2734-2739.

31. Sekar, M. M.; Bloch, W.; St John, P. M. Comparative study of sequencedependent hybridization kinetics in solution and on microspheres. Nucleic acids research 2005, 33 (1), 366-375.

32. Hooyberghs, J.; Baiesi, M.; Ferrantini, A.; Carlon, E. Breakdown of thermodynamic equilibrium for DNA hybridization in microarrays. Physical Review E 2010, 81 (1), 012901.

33. Medalion, S.; Wagman, M.; Grosberg, A.; Rabin, Y. Network Formation by Cross-Hybridization of Complementary Strands to Grafted ssDNA. ACS Macro Letters 2014, 3, 191-193.

34. Schmitt, T. J.; Knotts IV, T. A. Thermodynamics of DNA hybridization on surfaces. The Journal of chemical physics 2011, 134 (20), 205105.

35. Naiser, T.; Kayser, J.; Mai, T.; Michel, W.; Ott, A. Stability of a Surface-Bound Oligonucleotide Duplex Inferred from Molecular Dynamics: A Study of Single Nucleotide Defects Using DNA Microarrays. Physical Review Letters 2009, 102 (21), 218301. 
36. Ouldridge, T. E.; Šulc, P.; Romano, F.; Doye, J. P. K.; Louis, A. A. DNA hybridization kinetics: zippering, internal displacement and sequence dependence. Nucleic Acids Research 2013.

37. Sambriski, E. J.; Schwartz, D. C.; de Pablo, J. J. Uncovering pathways in DNA oligonucleotide hybridization via transition state analysis. Proceedings of the National Academy of Sciences 2009, 106 (43), 18125-18130.

38. Hoefert, M. J.; Sambriski, E. J.; Jose de Pablo, J. Molecular pathways in DNADNA hybridization of surface-bound oligonucleotides. Soft Matter 2011, 7 (2), 560566.

39. Stillman, B. A.; Tonkinson, J. L. Expression Microarray Hybridization Kinetics Depend on Length of the Immobilized DNA but Are Independent of Immobilization Substrate. Analytical Biochemistry 2001, 295 (2), 149-157.

40. Riccelli, P.; Merante, F.; Leung, K.; Bortolin, S.; Zastawny, R.; Janeczko, R.; Benight, A. S. Hybridization of single-stranded DNA targets to immobilized complementary DNA probes: comparison of hairpin versus linear capture probes. Nucleic acids research 2001, 29 (4), 996-1004.

41. Bishop, J.; Wilson, C.; Chagovetz, A. M.; Blair, S. Competitive Displacement of DNA during Surface Hybridization. Biophysical Journal 2007, 92 (1), L10-L12.

42. Zhang, N.-H.; Shan, J.-Y. An energy model for nanomechanical deflection of cantilever-DNA chip. Journal of the Mechanics and Physics of Solids 2008, 56 (6), 2328-2337.

43. Zhao, Y.; Ganapathysubramanian, B.; Shrotriya, P. Cantilever deflection associated with hybridization of monomolecular DNA film. Journal of Applied Physics 2012, $111(7)$, -.

44. Zhang, N.-H.; Meng, W.-L.; Tan, Z.-Q. A multi-scale model for the analysis of the inhomogeneity of elastic properties of DNA biofilm on microcantilevers. Biomaterials 2013, 34 (7), 1833-1842.

45. Calleja, M.; Carrascosa, L. G.; Tarin, A.; Tamayo, J. Study of the adsorption of sulfur-derivatized single stranded DNA on gold by atomic force microscopy and the cantilever bending technique. Sensor Letters 2006, 4 (3), 275-280.

46. Binder, H.; Preibisch, S. Specific and Nonspecific Hybridization of Oligonucleotide Probes on Microarrays. Biophysical Journal 2005, 89 (1), 337-352.

47. Schreiner, S. M.; Shudy, D. F.; Hatch, A. L.; Opdahl, A.; Whitman, L. J.; Petrovykh, D. Y. Controlled and Efficient Hybridization Achieved with DNA Probes Immobilized Solely through Preferential DNA-Substrate Interactions. Analytical Chemistry 2010, 82 (7), 2803-2810.

48. Yuan, M.; Zhan, S.; Zhou, X.; Liu, Y.; Feng, L.; Lin, Y.; Zhang, Z.; Hu, J. A Method for Removing Self-Assembled Monolayers on Gold. Langmuir 2008, 24 (16), 8707-8710.

49. Ansar, S. M.; Ameer, F. S.; Hu, W.; Zou, S.; Pittman, C. U.; Zhang, D. Removal of Molecular Adsorbates on Gold Nanoparticles Using Sodium Borohydride in Water. Nano Letters 2013, 13 (3), 1226-1229.

50. Martínez, N.; Kosaka, P. M.; Tamayo, J.; Ramírez, J.; Ahumada, O.; Mertens, J.; Hien, T.; Rijn, C.; Calleja, M. High throughput optical readout of dense arrays of nanomechanical systems for sensing applications. Review of Scientific Instruments 2010, 81 (12), 125109-125109-9. 
